\title{
Retiform Purpura In The Setting of COVID-19: A Harbinger of Underlying Coagulopathy and Severe Disease Course
}

\author{
Gabrielle Brody, BS ${ }^{1}$, Michael O. Nguyen, MD, $\mathrm{PhD}^{2}$, Delila Pouldar Foulad, $\mathrm{MD}^{2}$, Nathan W. \\ Rojek, MD ${ }^{2}$ \\ ${ }^{1}$ University of California, Irvine, School of Medicine, Irvine, CA \\ ${ }^{2}$ University of California, Irvine, Department of Dermatology, Irvine, CA
}

\section{ABSTRACT}

While the majority of COVID-19 cases are mild and can be managed in the outpatient setting, more severe cases have proven to be a clinical challenge. While some cases demonstrate a more slow and indolent decline, others seem to deteriorate rapidly with little forewarning. The current literature has connected Retiform Purpura as a cutaneous manifestation associated with severe COVID-19 infections, however timing of cutaneous presentation and severe clinical COVID-19 symptoms has not been well described. Here we report a case of a 58-year-old female who developed Retiform Purpura nearly a week prior to the development of any significant COVID-19 symptoms. This case demonstrates that Retiform Purpura is not only associated with severe COVID-19 disease, but can present prior to symptom onset and should be seen as a harbinger for impending clinical deterioration.

\section{INTRODUCTION}

While COVID-19 is most known for its respiratory presentation, other organ systems are by no means unscathed. Here, we report a case of retiform purpura associated with COVID-19 pneumonia and highlight how retiform purpura may be a useful indicator for future clinical course.

\section{CASE REPORT}

A 58-year-old otherwise healthy woman presented to the emergency department in acute hypoxic respiratory distress secondary to COVID-19 pneumonia diagnosed ten days prior at an outside care facility. One day prior to admission, she developed significant shortness of breath and required home oxygen. As her pulmonary status further declined, she presented to the emergency department with an oxygen saturation of $79 \%$. Nasopharyngeal swab for PCR testing of COVID-19 confirmed active infection. The patient had not received any COVID-19 vaccines. Labs were notable for elevations in D-dimer $(3,160 \mathrm{ng} / \mathrm{ml}), \quad$ C-reactive protein $(10 \mathrm{mg} / \mathrm{dl})$, lactate dehydrogenase $(569 \mathrm{U} / \mathrm{L})$, and troponin $(26 \mathrm{ng} / \mathrm{L})$, correlating with severe COVID-19 infection. ${ }^{1}$ Chest x-ray revealed diffuse opacities consistent with COVID-19 pneumonia and initial CTA was negative for pulmonary embolism.

Physical exam was significant for an exquisitely tender, violaceous, stellate plaque with central necrosis on the right hip (Fig. 1), which was first noticed six days prior to presentation. Notably, she had no medication exposures except for longstanding vitamin $\mathrm{C}$ and vitamin $D$ supplementation, no recent travel, and no signs concerning for either July 2021 Volume 5 Issue 4 
malignancy or connective tissue disease. Her findings were determined to be clinically consistent with retiform purpura secondary to COVID-19 infection. Prophylactic anticoagulation with enoxaparin $40 \mathrm{mg}$ was initiated.

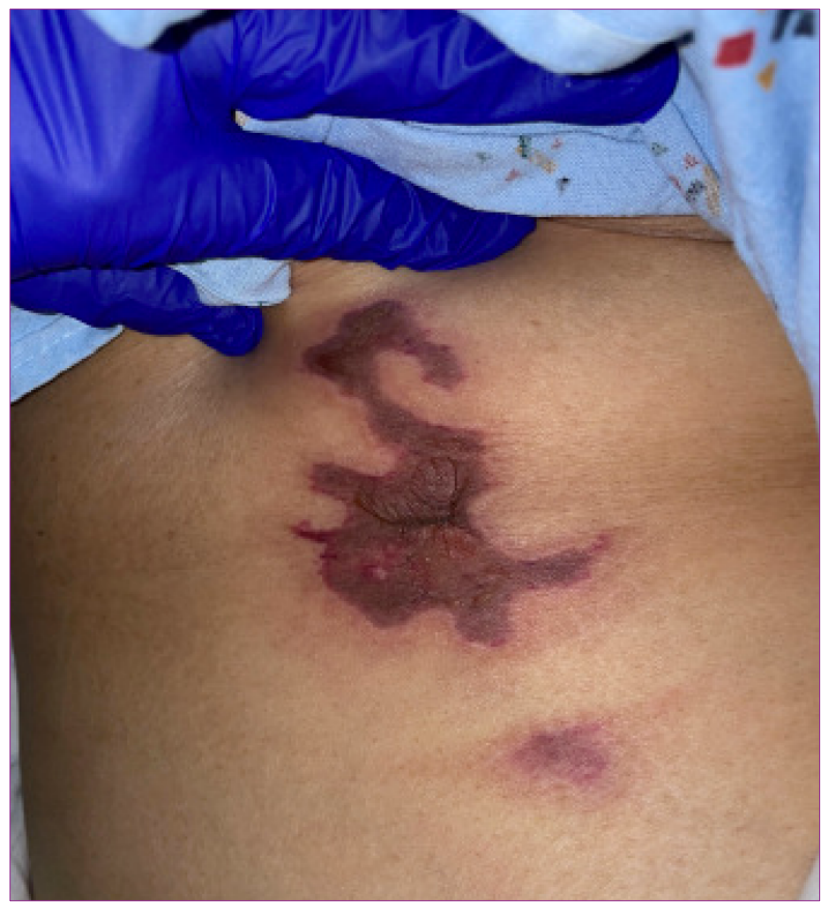

Figure 1. Violaceous stellate plaque with central necrosis on the right lateral hip, consistent with retiform purpura on day of admission.

On the fifth day of admission, the patient developed worsening pleuritic chest pain, hypoxia and cough. Additional labs revealed an increased D-dimer from 3,160 to $>20,000$ $\mathrm{ng} / \mathrm{mL}$, repeat CTA demonstrated new multiple segmental and subsegmental pulmonary emboli, and lower extremity ultrasound with Doppler showed a nonocclusive right popliteal vein thrombus.

The patient was discharged on hospital day eight on six liters of oxygen, a ten-day course of dexamethasone, and an extended course of therapeutic anticoagulation with apixaban. Two weeks after discharge, the patient was reassessed and the retiform purpuric plaque had progressed with expected central necrotic ulceration (Fig. 2). During this time course, she did not develop any other new cutaneous lesions.

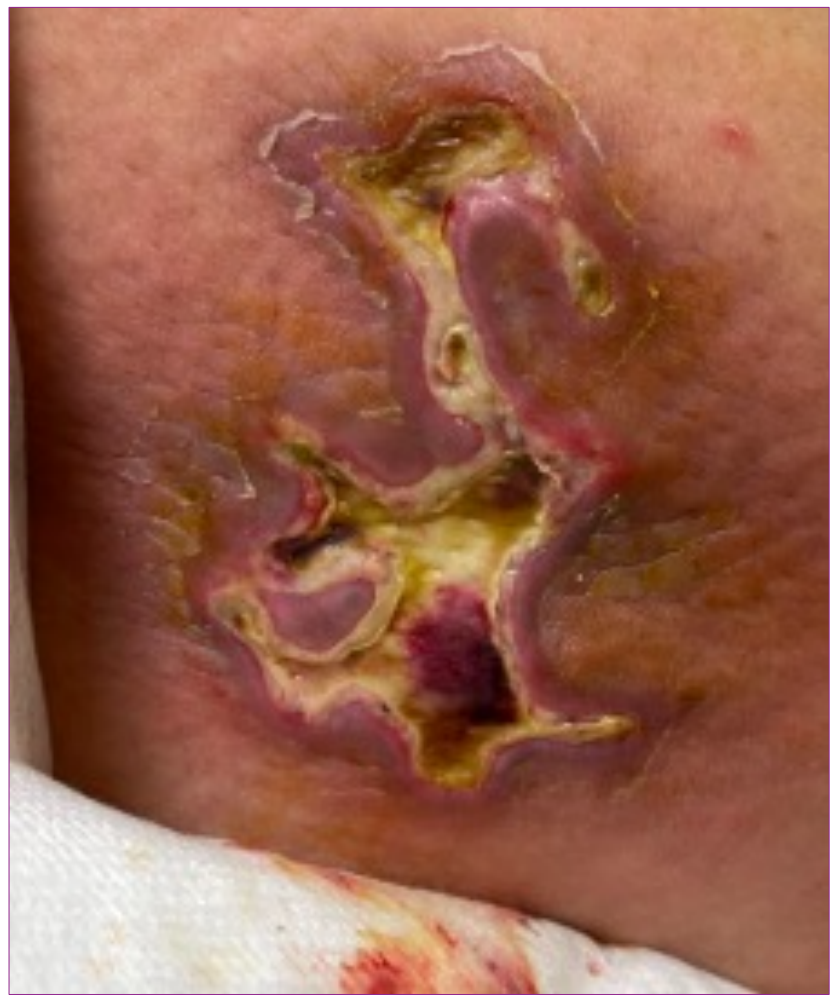

Figure 2. Progression of retiform purpura with central necrotic ulceration on right lateral hip two weeks after discharge.

\section{DISCUSSION}

There is a vast array of dermatologic manifestations reported in association with COVID-19, which are grouped into seven morphologic categories: morbilliform, perniolike, urticarial, macular erythema, vesicular, papulosquamous, and retiform purpura. ${ }^{2}$ Retiform purpura is one of the least represented dermatologic manifestations, accounting for just $6.4 \%$ of cases, and corresponds with severe COVID-19 infection. ${ }^{2}$ A recent study by Conforti et al reported that of 11 patients with COVID-19 and retiform purpura, $100 \%$ were hospitalized, $82 \%$ developed acute respiratory distress and $91 \%$ required ECMO 
and/or ventilation. ${ }^{2}$ The reason for its association with high disease burden is still being elucidated. Magro et al. noted that patients with pernio-like lesions secondary to COVID-19 who have mild respiratory symptoms and good outcomes have a strong inflammatory response with low viral loads, while patients with retiform purpura with severe COVID-19 disease have minimal interferon response, high viral loads and profound vasculopathy. It is hypothesized that this difference in microenvironment possibly allows for unchecked viral replication and rampant inflammatory activation. $^{3}$

\section{CONCLUSION}

This case highlights the seriousness of retiform purpura in COVID-19 patients as a cutaneous finding concerning for an underlying vasculopathy that may precede further acute thromboembolic events and a worsening course of disease. The presence of retiform purpura in patients with COVID-19 therefore merits close clinical monitoring for the risk of acute decompensation and discussion of the benefits and risks of anticoagulant therapy for optimizing outcomes.

\section{Conflict of Interest Disclosures: None}

Funding: None

Corresponding Author:

Gabrielle Brody, BS

843 Health Sciences Road

Hewitt Hall 1001

Irvine, CA 92697

Email: gabbynbrody@gmail.com
2. Freeman EE, McMahon DE, Lipoff JB, et al. The spectrum of COVID-19-associated dermatologic manifestations: An international registry of 716 patients from 31 countries. J Am Acad Dermatol. 2020;83(4):1118-1129. doi:10.1016/j.jaad.2020.06.1016

3. Magro C, Mulvey J, Laurence J, et al. The differing pathophysiologies that underlie COVID-19associated perniosis and thrombotic retiform purpura: a case series Conflicts of interest. $\mathrm{Br} \mathrm{J}$ Dermatol. 2020. doi:10.1111/bjd.19415

\section{References:}

1. Velavan TP, Meyer CG. Mild versus severe COVID-19: Laboratory markers. Int J Infect Dis. 2020;95:304-307. doi:10.1016/j.jijid.2020.04.061 